\title{
Significance of stromal decorin expression during the progression of breast cancer
}

\author{
GOSHI ODA $^{1}$, TAKANOBU SATO ${ }^{1}$, TOSHIAKI ISHIKAWA ${ }^{2}$, HIROSHI KAWACHI ${ }^{3}$, TSUYOSHI NAKAGAWA ${ }^{1}$, \\ TAKASHI KUWAYAMA ${ }^{1}$, MEGUMI ISHIGURO ${ }^{2}$, SATORU IIDA ${ }^{1}$, HIROYUKI UETAKE ${ }^{2}$ and KENICHI SUGIHARA ${ }^{1}$ \\ Departments of ${ }^{1}$ Surgical Oncology, ${ }^{2}$ Translational Oncology, and ${ }^{3}$ Pathology, Tokyo Medical and \\ Dental University, Graduate School of Medicine and Dentistry, Bunkyo-ku, Tokyo 113-8519, Japan
}

Received July 12, 2012; Accepted August 27, 2012

DOI: $10.3892 /$ or.2012.2040

\begin{abstract}
Decorin, a small leucine-rich proteoglycan and important component of the extracellular matrix (ECM), is a natural anticancer agent that modulates several receptors involved in cell growth and survival. Reductions in decorin expression may weaken the ECM and enhance the effectiveness of these receptors and may, consequently, lead to tumor spreading. To determine the contribution of stromal decorin regulation in the development of breast cancer and in tumor invasiveness, immunohistochemistry was used to examine the expression of stromal decorin in 120 breast cancer tissue samples. In patients with invasive breast carcinoma (IBC), stromal decorin expression was highest in normal gland tissue, lower in in situ components and the lowest in invasive components. Stromal decorin expression adjacent to malignant cells in IBC tumors was also significantly weaker compared to that in pure ductal carcinoma in situ (DCIS). These findings indicate that there is a striking difference in the stromal decorin expression around normal glands and around DCIS or IBC tumors. Reduced levels of decorin were associated with more aggressive disease; this finding was consistent with the view that reduced decorin expression may facilitate tumorigenesis, tumor invasion and/or tumor growth. Given these and other reported findings, evaluating stromal decorin expression may
\end{abstract}

Correspondence to: Dr Toshiaki Ishikawa, Department of Translational Oncology, Tokyo Medical and Dental University, Graduate School of Medicine and Dentistry, 1-5-45 Yushima, Bunkyo-ku, Tokyo 113-8519, Japan

E-mail: ishi.srg2@tmd.ac.jp

Abbreviations: DCIS, ductal carcinoma in situ; IBC, invasive breast carcinoma; ECM, extracellular matrix; TGF- $\beta$, transforming growth factor- $\beta$; EGFR, epidermal growth factor receptor; IGF-IR, insulin-like growth factor receptor; LRP, low-density lipoprotein receptor-related protein; $\mathrm{MW}$, microwave; FEA, flat epithelial atypia; $\mathrm{ADH}$, atypical ductal hyperplasia; ER, expression of estrogen receptor; $\mathrm{PgR}$, progesterone receptor; MMPs, matrix metalloproteinases

Key words: decorin, proteoglycan, breast cancer, extracellular matrix, cancer stroma be useful in assessing prognosis and malignant potential; therefore, a large-scale study of decorin expression is warranted.

\section{Introduction}

The extracellular matrix (ECM), which provides structural support to cells, is the extracellular material in tissue that generally has a role in the regulation of cell proliferation, differentiation and wound healing. Components of the ECM are now being recognized as key signaling molecules that affect the invasion and metastasis of cancer. It has become evident that the ECM modulates cellular proliferation and differentiation by affecting not only growth factors, but also various receptors involved in controlling morphogenesis and cell growth (1). Decorin is a component of the ECM that is synthesized primarily by fibroblasts and myofibroblasts (2) and that regulates collagen fibrillogenesis $(3,4)$. Based on in vitro assays, decorin is a potent inhibitor of tumor cell proliferation as it interacts with transforming growth factor- $\beta$ (TGF- $\beta$ ) (5) and affects several receptors, epidermal growth factor receptor (EGFR), insulin-like growth factor receptor (IGF-IR) and lowdensity lipoprotein receptor-related protein (LRP) (2). Evidence from in vitro experiments indicates that decorin is a potent inhibitor of tumor cell proliferation, therefore, the antitumor effects of decorin have been tested in vivo. A cytomegaloviral vector containing the decorin transgene has been shown to inhibit tumorigenesis and metastatic spreading of breast carcinoma (6). A recombinant protein that comprises only the core of the human decorin protein inhibits metastatic spreading to the lung in xenograft models of breast carcinoma (7).

The natural history of breast cancer involves a progression that begins with abnormal proliferation of epithelial cells, which can progress to ductal carcinoma in situ (DCIS), which in turn can become invasive breast carcinoma (IBC). This progression can culminate in metastatic disease (8). Cancer invasion is the process in which malignant cells break away from a primary tumor and spread through surrounding tissue by interaction with components of the stroma. The development of invasiveness is a critical event for patients since malignant cells acquire the capacity to metastasize during this process. The mechanisms by which primary breast cancer cells acquire an invasive phenotype and break the basal membrane are not fully understood. 
During invasion, the stroma is altered by interactions with breast cancer cells, and the stromal environment becomes susceptible to invasion. Changes in the stroma may occur at the early stage of the transition from DCIS to IBC. Several of the critical changes in the tumor stroma that accompany cancer progression have yet to be identified. To identify critical changes in the stroma that promote invasion, we focused on changes in the expression of decorin which has anticancer effects and is a component of ECM. In particular, we hypothesized that expression of decorin in the stroma may decrease during the development and progression of breast cancer. To test this hypothesis, we examined decorin expression in the stroma of tissue samples in different histological categories [normal glands, flat epithelial atypia (FEA), atypical ductal hyperplasia (ADH), in situ component, invasive component] in individual IBC patients. We also compared pure DCIS samples to IBC samples.

This is the first study to demonstrate that stromal decorin expression decreases during the progression of breast cancer. Reduced decorin expression may facilitate tumorigenesis, tumor invasion and/or tumor growth.

\section{Materials and methods}

Patients and tissue samples. We obtained 120 tumor samples: 98 were from patients with IBC and 22 were from patients with DCIS. All patients underwent surgical resection in the Department of Breast Surgery at Tokyo Medical and Dental University, Japan, between January 1999 and November 2003. Mean patient age was 54.6 years (range, 30-91 years). None of the patients had distant metastasis. All specimens were formalin-fixed and paraffin-embedded. The Institutional Review Board approved the study, and written informed consent was obtained from each patient before surgery.

Examination of clinicopathological and biological features. After tissue samples were stained with hematoxylin-eosin (H\&E), histopathological examination was performed using the International Union Against Cancer Tumor-NodeMetastasis classification criteria (9). Expression of estrogen receptor $(\mathrm{ER})$ and progesterone receptor $(\mathrm{PgR})$ were evaluated using immunohistochemistry. The status of each tumor with regard to ER and $\mathrm{PgR}$ expression was determined by calculating the percentage of all cancer cells within a given tumor with positive nuclear staining; the cut-off value was set at $10 \%$.

Decorin immunohistochemical staining. For immunohistochemical analyses, tissue sections $(4-\mu \mathrm{m})$ were deparaffinized over the course of five 10-min incubations in xylene. Tissue sections were rehydrated, and antigen retrieval was then performed by incubating the sections in $10 \mathrm{mmol} / 1$ sodium citrate buffer ( $\mathrm{pH}$ 6.0) in a temperature-controllable microwave (MW) processor (MI-77; Azumaya Co., Tokyo, Japan) at $98^{\circ} \mathrm{C}$ for $25 \mathrm{~min}$. Endogenous peroxidase activity was blocked using a solution of $3 \%$ hydrogen peroxide in absolute methanol for $15 \mathrm{~min}$. Sections were incubated with anti-decorin mouse monoclonal antibodies (ab54728, Abcam, Cambridge, UK) (1:1,000 dilution); the sections were then beam irradiated with the MW processor at $27^{\circ} \mathrm{C}$ for $15 \mathrm{~min}$. The Histofine SAB-PO kit (Nichirei Corp., Tokyo, Japan) was used according to the manufacturer's instructions to block non-specific binding and to detect bound primary antibody. Color development was carried out with DAB (0.02\% 3-3'-diaminobenzidine tetrahydrochloride; Nichirei) for $10 \mathrm{~min}$ at room temperature. The sections were then counterstained with Mayer's hematoxylin.

Immunohistochemical evaluation. The immunostaining of decorin was analyzed under a light microscope. The evaluation of stromal decorin expression was performed around normal gland tissue, pre-malignant components and in situ components. In the cases of IBC, the stroma adjacent to malignant cells was evaluated. First, areas of tissue that represented each of the different histological categories (normal gland, FEA, ADH, in situ component, invasive component) within samples from each individual IBC patient were evaluated separately. Additionally, samples from patients with pure DCIS were compared to those from patients with IBC. We performed semiquantitative digital image analysis. The intensity of decorin signal was evaluated using the ImageJ software (version 1.43u, National Institutes of Health, Bethesda, MD, USA), according to the method described by Augoff et al (10). Briefly, stained specimens were viewed using a light microscope, and random areas at the periphery of lesions were captured as digital images (680x512 pixels) with a digital camera. For each digital image, the signal from 10 representative areas was digitized into a grayscale ranging from 0 (white) to 255 (black), and these data were used to generate a histogram. Cellular nuclei were omitted from this analysis since they were counterstained with hematoxylin, which would have artificially increased the gray-level values. The stroma in the negative control samples (samples without primary antibody) was used as an internal control. The intensity of decorin signal was standardized by subtracting the mean gray value of the internal control.

Statistical analysis. The Wilcoxon signed-rank test was used for comparisons of different histological categories within individual IBC patients. Quantitative decorin expression for comparison of IBC and pure DCIS were analyzed using the Mann-Whitney U test. The Mann-Whitney U test was also used to examine the association between decorin expression and clinicopathological/biological features. P-values $<5 \%$ were considered to indicate statistically significant differences.

\section{Results}

Decorin expression. Decorin was present mainly in the ECM and was clearly expressed by stromal cells, such as fibroblasts and some inflammatory cells. Decorin expression was also evident around normal gland tissue and the in situ component (Fig. 1). However, in some cases, the decorin expression in the stroma around the in situ component was weak. Decorin expression in stroma tended to be weaker in the invasive components than in the in situ components (Fig. 2). Decorin signals were almost completely absent from epithelial cells.

Initially, we compared different histological categories (normal glands, FEA, ADH, in situ component, invasive component) in individual IBC patients. When a single IBC had regions with different histological features, the stromal decorin expression around each feature was evaluated sepa- 

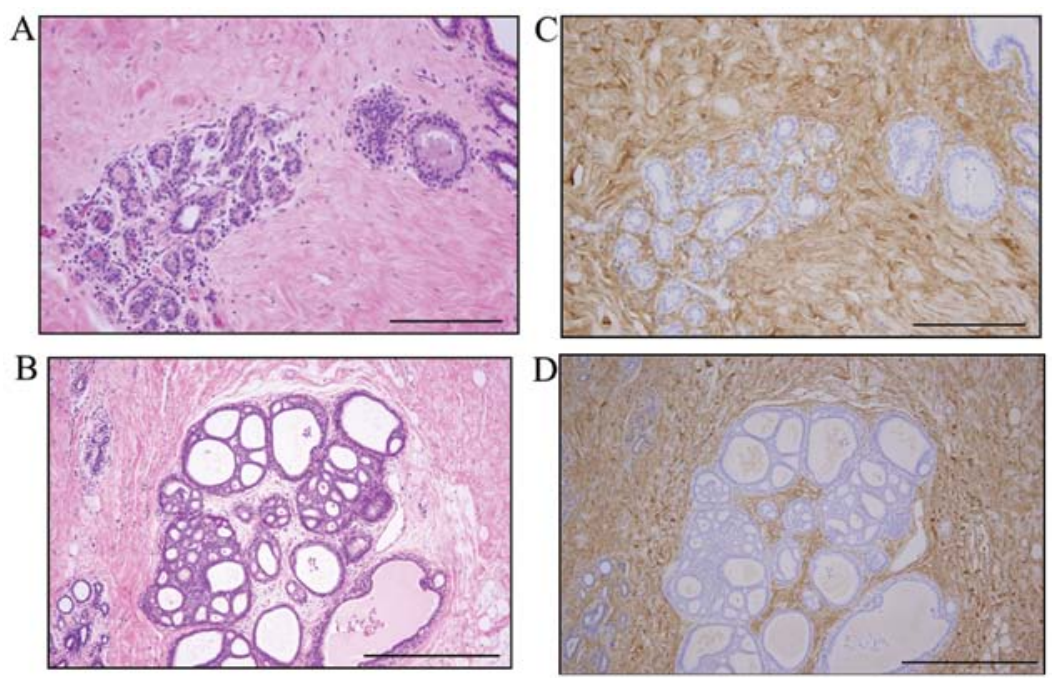

Figure 1. Immunohistochemical staining of decorin expression in normal gland tissue and DCIS. Decorin signal was mainly present in the ECM, fibroblasts and some inflammatory cells. Decorin signal was almost completely absent from epithelial cells. (A) Normal gland (H\&E; bar, $200 \mu \mathrm{m})$. (B) DCIS (H\&E; bar, $500 \mu \mathrm{m}$ ). (C) Stromal decorin expression around normal gland tissue. The serial section is the same as that in (A) (bar, $200 \mu \mathrm{m}$ ). (D) Stromal decorin expression around DCIS. The serial section is the same as that in (B) (bar, $500 \mu \mathrm{m}$ ).
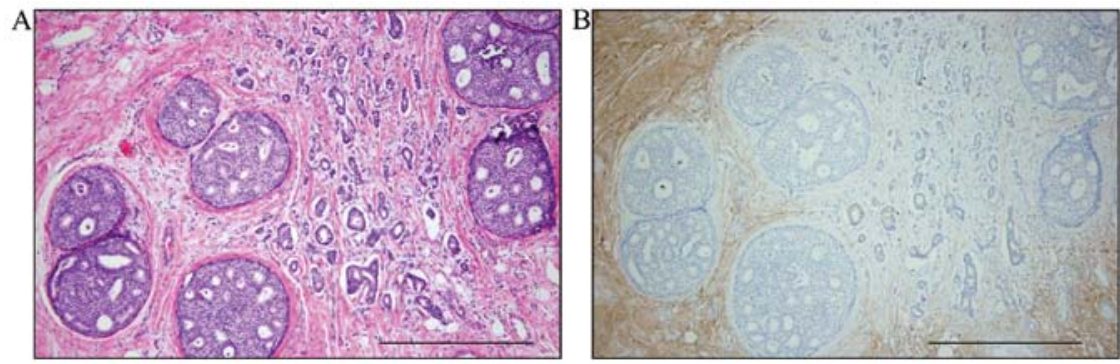

Figure 2. Immunohistochemical staining of decorin in invasive breast carcinoma with in situ component. This tumor has an invasive component between in situ components. (A) H\&E (bar, $500 \mu \mathrm{m}$ ). (B) A serial section from the same tissue block. Stromal decorin expression in the invasive component was weaker than that in the in situ component (bar, $500 \mu \mathrm{m}$ ).

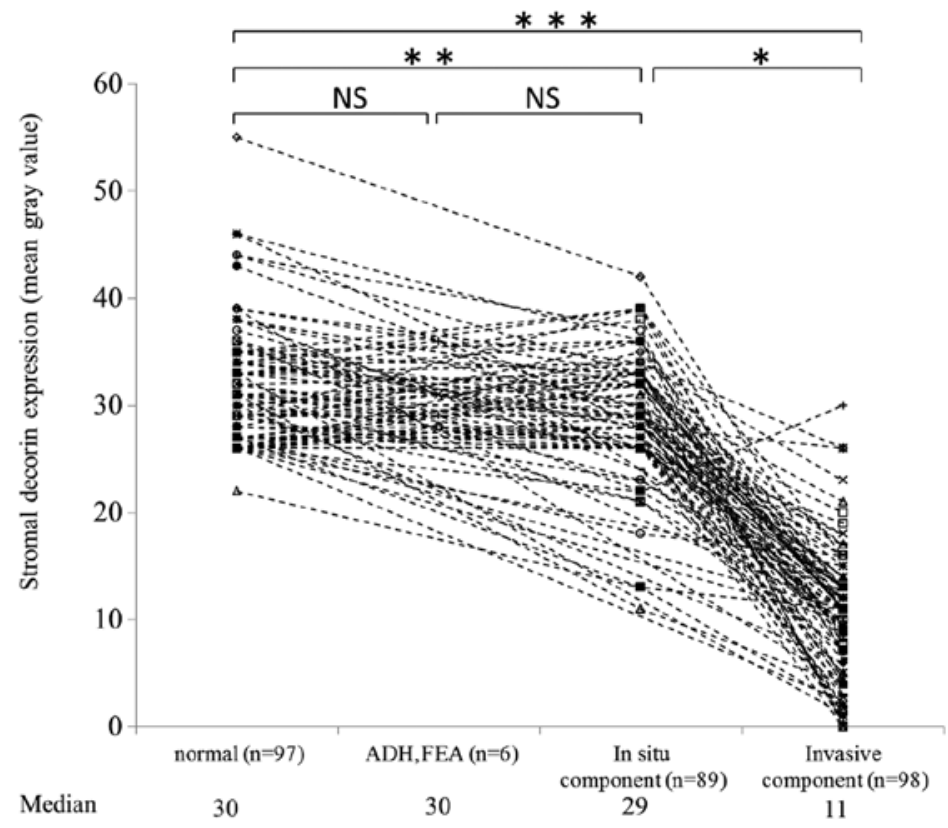

Figure 3. Comparison of stromal decorin levels around normal glands, atypical ductal hyperplasia (ADH), flat epithlial atypia (FEA), in situ component and invasive component within individual IBC patients. Each dashed line connects the data points that derived from one patient. Mean gray value was estimated using ImageJ software (version 1.44, National Institutes of Health). P-values were estimated by the Wilcoxon signed-rank test. P $<0.05$ denoted statistically significant differences. ${ }^{*} \mathrm{P}<0.0001,{ }^{* *} \mathrm{P}=0.001,{ }^{* * *} \mathrm{P}<0.0001 ; \mathrm{NS}$, not significant. 

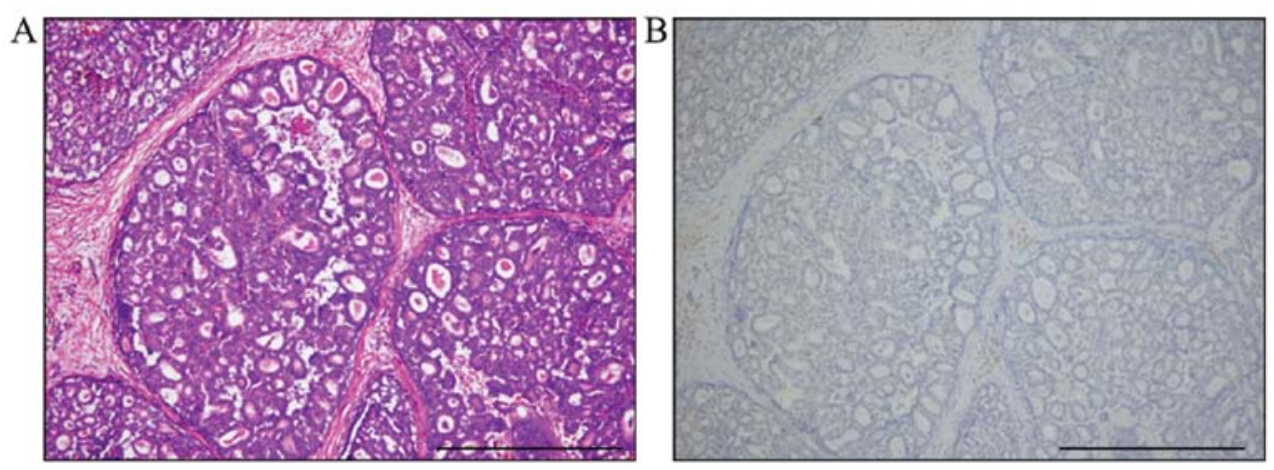

Figure 4. Weak stromal decorin expression around DCIS. (A) H\&E (bar, $500 \mu \mathrm{m}$ ). (B) A serial section from the same tissue block. Stromal decorin expression around the DCIS tissue was weak (bar, $500 \mu \mathrm{m}$ ).

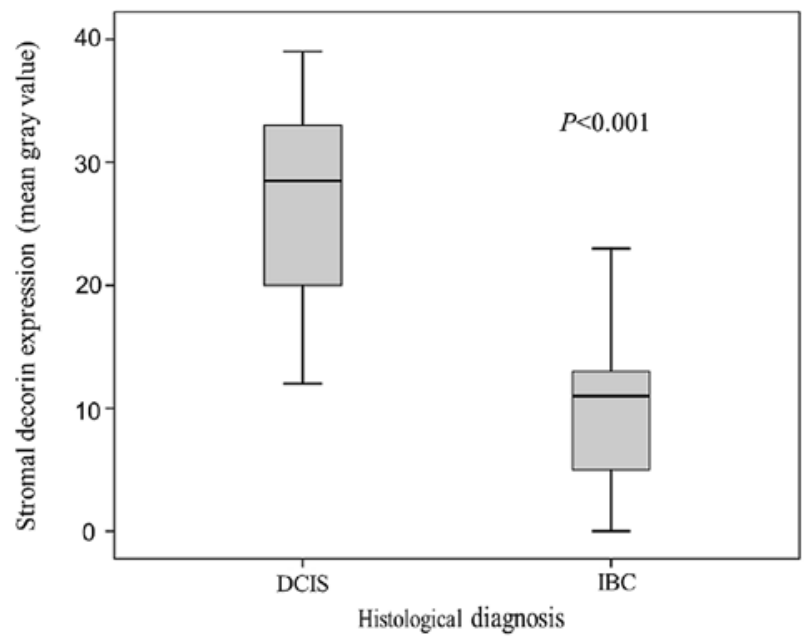

Figure 5. Comparison of stromal decorin expression in pure DCIS to that in IBC. Mean gray value was estimated using ImageJ software. P-values were determined using the Mann-Whitney $\mathrm{U}$ test; $\mathrm{P}<0.05$ was considered statistically significant. Stromal decorin expression adjacent to malignant cells in IBC was lower than that in pure DCIS.

rately; these results are shown in Fig. 3. Stromal decorin expression was significantly lower in invasive components than in the other components. Stromal decorin expression was also lower in the in situ components than in normal glands, FEA, or ADH.

Subsequently, we compared pure DCIS samples to IBC samples. The mean value of stromal decorin expression around DCIS (in situ component) was $27 \pm 8$.1. In 6 cases of DCIS, very weak decorin staining in the stroma was detected (Fig. 4). In IBC patients, the mean value of stromal decorin expression around invasive components was $10 \pm 6.1$. The values of decorin expression were significantly different in DCIS and IBC samples ( $\mathrm{P}<0.001$, Fig. 5).

Decorin expression and clinicopathological/biological features. Correlations between stromal decorin expression adjacent to malignant cells and clinicopathological/biological features are summarized in Table I. Stromal decorin expression was significantly lower in IBC samples than in DCIS samples. There was no statistically significant correlation between stromal decorin expression and age, breast density, menopausal status, tumor location or hormone receptor expression.

\section{Discussion}

This is the first immunohistochemistry study to thoroughly assess decorin expression around normal glands, ductal carcinoma in situ (DCIS) and invasive breast carcinoma (IBC). We showed direct evidence that stromal decorin expression was highest in normal tissue, lower in in situ components and the lowest in invasive components. We also demonstrated that stromal decorin expression was significantly lower in cancer tissues from patients with IBC than in cancer tissues from patients with DCIS.

Our results indicate the possibility that downregulation of stromal decorin expression may be involved in the progression of breast cancer. Similar results have been reported in other types of cancer. In colorectal tumors, the stromal decorin of adenocarcinoma tends to have significantly lower expression than normal tissue and adenoma (11). These results support the hypothesis that the downregulation of decorin expression is associated with cancer progression and carcinogenesis. However, to date, there have been no detailed immunohistochemical studies on decorin expression in breast cancer.

The biological implications of decreased decorin expression with respect to disease aggressiveness have yet to be determined. One explanation of the reduced decorin expression is that a reduction in decorin expression might weaken the functional and physical barriers to tumor invasion since decorin plays an important role in maintaining normal collagen structure (12). A second possible explanation is that decreased decorin expression might allow for the accumulation of active TGF- $\beta$. Cell growth inhibition is reported to be due to the ability of decorin to bind TGF- $\beta(5,13)$. Although there are multiple opinions on this issue, TGF- $\beta$ may actually be stimulatory at early stages of epithelial tumorigenesis $(14,15)$. A third possibility is that low levels of stromal decorin might promote tumor invasion by affecting the signaling pathways associated with EGFR and other Erb-B family receptors. Decorin protein is a biological ligand of EGFR, but its interaction with EGFR is different from that of other typical ligands (16). Decorin binding to EGFR leads to transient activation of the receptor tyrosine kinase, and this activation is followed by the phos- 
Table I. Stromal decorin expression and clinicopathological/biological features.

\begin{tabular}{|c|c|c|c|}
\hline Characteristics & $\begin{array}{l}\text { No. of patients } \\
\quad(\mathrm{n}=120)\end{array}$ & $\begin{array}{l}\text { Stromal decorin expression } \\
\text { (median gray value) }\end{array}$ & P-value \\
\hline \multicolumn{4}{|l|}{ Age } \\
\hline$\leq 50$ & 50 & 12 & \\
\hline$>50$ & 70 & 11 & NS \\
\hline \multicolumn{4}{|l|}{ Breast density ${ }^{\mathrm{a}}$} \\
\hline Low & 70 & 11 & \\
\hline High & 50 & 12 & NS \\
\hline \multicolumn{4}{|l|}{ Menopausal status } \\
\hline Pre-menopausal & 65 & 12 & \\
\hline Post-menopausal & 55 & 11 & NS \\
\hline \multicolumn{4}{|l|}{ Tumor site } \\
\hline Inner & 44 & 13 & \\
\hline Outer & 76 & 11 & NS \\
\hline \multicolumn{4}{|l|}{ ER } \\
\hline Positive ( $\geq 10 \%$ ) & 77 & 12 & \\
\hline Negative & 43 & 11 & NS \\
\hline \multicolumn{4}{|l|}{ PgR } \\
\hline Positive ( $\geq 10 \%$ ) & 70 & 12 & \\
\hline Negative & 50 & 11 & NS \\
\hline \multicolumn{4}{|c|}{ Histological diagnosis } \\
\hline DCIS & 22 & 28.5 & \\
\hline IBC & 98 & 11 & $<0.001$ \\
\hline
\end{tabular}

${ }^{a}$ When a percentage of the breast area is $>50 \%$, breast density is defined as high. P-values were estimated by the $\chi^{2}$ and Fisher's exact probability test. ER, estrogen receptor; PgR, progesterone receptor; DCIS, ductal carcinoma in situ; IBC, invasive breast carcinoma; NS, not significant.

phorylation of MAP kinase, the induction of p21 and growth suppression $(16,17)$.

The mechanisms that cause downregulation of stromal decorin are not known. Production of decorin from myofibroblasts and fibroblasts around the periphery of the cancerous tissue might decrease. Decorin originates from stromal fibroblasts and myofibroblasts (2), but there is no evidence that cancer-associated-fibroblasts produce decorin. Alternatively or additionally, matrix metalloproteinases (MMPs), which are secreted by breast carcinoma or stromal cells (18), may cleave the decorin around the duct and thereby accelerate cancer invasion. Decorin is reported to be degraded by MMP-2, -3, and -7 (19) and MMP-2 and MMP-7 are expressed in breast carcinoma (20-23). As there is no direct evidence that MMP-2 or MMP-7 cleaves decorin in breast cancer, this question should be addressed directly in future studies.

We also demonstrated that stromal decorin expression was lower in the in situ component compared to normal glands, flat epithelial atypia (FEA) and atypical ductal hyperplasia (ADH). One possible explanation for this difference is that the stroma was altered and became favorable to cancer. Another possible explanation is that transformation of stroma before invasion might induce carcinogenesis. Quante et al suggested that, based on studies of mouse models, bone marrow-derived myofibroblasts contribute to the mesenchymal stem cell niche and promote tumor growth (24). In any case, decreased expression of decorin around DCIS may represent a local, negative host response that contributes to invasion of cancer.

Based on PCR and western blot studies, decorin expression and prognostic factors of malignant tumors are related. For example, stromal decorin expression is reduced in soft tissue sarcoma and reduction of decorin expression is associated with poor outcomes (25). In breast cancer, reduced decorin expression in the peritumoral stroma of breast cancer worsens the prognosis in node-negative patients (26). However, to date, there have been no detailed immunohistochemical studies on decorin expression in breast cancer. Further immunohistochemical studies are required to fully assess the relationship between prognosis and stromal decorin expression.

Systemic injection of a recombinant protein comprising the decorin core protein can reduce breast tumor growth and halt metastatic spread to the lungs (7). Decorin may play an important role for the treatment of breast cancer.

In conclusion, we have shown that the stromal decorin expression around DCIS or IBC tumors is markedly different from that in normal gland tissue. Reduced decorin expression may facilitate tumorigenesis, tumor invasion and/or tumor growth. A future study to confirm and further assess the prognostic significance of stromal decorin expression in breast carcinoma is warranted. 


\section{Acknowledgements}

The authors thank Y. Takagi for excellent technical assistance.

\section{References}

1. Kim SH, Turnbull $\mathbf{J}$ and Guimond S: Extracellular matrix and cell signalling: the dynamic cooperation of integrin, proteoglycan and growth factor receptor. J Endocrinol 209: 139-151, 2011.

2. Goldoni S and Iozzo R: Tumor microenvironment: Modulation by decorin and related molecules harboring leucine-rich tandem motifs. Int J Cancer 123: 2473-2479, 2008.

3. Iozzo R: Matrix proteoglycans: from molecular design to cellular function. Annu Rev Biochem 67: 609-652, 1998.

4. Reed CC and Iozzo RV: The role of decorin in collagen fibrillogenesis and skin homeostasis. Glycoconj J 19: 249-255, 2002.

5. Yamaguchi Y, Mann D and Ruoslahti E: Negative regulation of transforming growth factor-beta by the proteoglycan decorin. Nature 346: 281-284, 1990

6. Araki K, Wakabayashi H, Shintani K, et al: Decorin suppresses bone metastasis in a breast cancer cell line. Oncology 77: 92-99, 2009.

7. Goldoni S, Seidler D, Heath J, et al: An antimetastatic role for decorin in breast cancer. Am J Pathol 173: 844-855, 2008.

8. Burstein HJ, Polyak K, Wong JS, Lester SC and Kaelin CM: Ductal carcinoma in situ of the breast. N Engl J Med 350: 1430-1441, 2004

9. Sobin LH and Wittekind C (eds): TNM Classification of Malignant Tumors. 6th edition. John Wiley \& Sons, New York, 2002 .

10. Augoff K, Grabowski K, Rabczynski J, Kolondra A, Tabola R and Sikorski A: Expression of decorin in esophageal cancer in relation to the expression of three isoforms of transforming growth factor-beta (TGF-beta1, -beta2, and -beta3) and matrix metalloproteinase-2 activity. Cancer Invest 27: 443-452, 2009.

11. Augoff K, Rabczynski J, Tabola R, Czapla L, Ratajczak K and Grabowski K: Immunohistochemical study of decorin expression in polyps and carcinomas of the colon. Med Sci Monit 14: CR530-CR535, 2008.

12. Ferdous Z, Wei V, Iozzo R, Höök M and Grande-Allen K: Decorin-transforming growth factor-interaction regulates matrix organization and mechanical characteristics of three-dimensional collagen matrices. J Biol Chem 282: 35887-35898, 2007.
13. Ständer M, Naumann U, Dumitrescu L, et al: Decorin gene transfer-mediated suppression of TGF-beta synthesis abrogates experimental malignant glioma growth in vivo. Gene Ther 5: 1187-1194, 1998

14. Reiss M and Barcellos-Hoff MH: Transforming growth factorbeta in breast cancer: a working hypothesis. Breast Cancer Res Treat 45: 81-95, 1997.

15. Akhurst RJ and Balmain A: Genetic events and the role of TGF beta in epithelial tumour progression. J Pathol 187: 82-90, 1999.

16. Iozzo R, Moscatello D, McQuillan D and Eichstetter I: Decorin is a biological ligand for the epidermal growth factor receptor. J Biol Chem 274: 4489-4492, 1999.

17. Csordás G, Santra M, Reed C, et al: Sustained down-regulation of the epidermal growth factor receptor by decorin. A mechanism for controlling tumor growth in vivo. J Biol Chem 275: 32879-32887, 2000.

18. Polette M, Gilbert N, Stas I, et al: Gelatinase A expression and localization in human breast cancers. An in situ hybridization study and immunohistochemical detection using confocal microscopy. Virchows Arch 424: 641-645, 1994.

19. Imai K, Hiramatsu A, Fukushima D, Pierschbacher $M$ and Okada Y: Degradation of decorin by matrix metalloproteinases: identification of the cleavage sites, kinetic analyses and transforming growth factor-beta1 release. Biochem J 322: 809-814, 1997.

20. Iwata H, Kobayashi S, Iwase H, Masaoka A, Fujimoto $\mathrm{N}$ and Okada Y: Production of matrix metalloproteinases and tissue inhibitors of metalloproteinases in human breast carcinomas. Jpn J Cancer Res 87: 602-611, 1996.

21. Jones JL, Glynn P and Walker RA: Expression of MMP-2 and MMP-9, their inhibitors, and the activator MT1-MMP in primary breast carcinomas. J Pathol 189: 161-168, 1999.

22. Garbett EA, Reed MW, Stephenson TJ and Brown NJ: Proteolysis in human breast cancer. Mol Pathol 53: 99-106, 2000.

23. Jiang WG, Davies G, Martin TA, et al: Targeting matrilysin and its impact on tumor growth in vivo: the potential implications in breast cancer therapy. Clin Cancer Res 11: 6012-6019, 2005.

24. Quante M, Tu SP, Tomita H, et al: Bone marrow-derived myofibroblasts contribute to the mesenchymal stem cell niche and promote tumor growth. Cancer Cell 19: 257-272, 2011.

25. Matsumine A, Shintani K, Kusuzaki K, et al: Expression of decorin, a small leucine-rich proteoglycan, as a prognostic factor in soft tissue tumors. J Surg Oncol 96: 411-418, 2007.

26. Troup S, Njue C, Kliewer E, et al: Reduced expression of the small leucine-rich proteoglycans, lumican, and decorin is associated with poor outcome in node-negative invasive breast cancer. Clin Cancer Res 9: 207-214, 2003. 